\title{
Efficient trapping of silver cations in a rare gas matrix: $\mathrm{Ag}_{3}^{+}$in argon
}

\author{
Sylvain Lecoultre, Alexandre Rydlo, and Christian Félix \\ Institut de Physique des Nanostructures, École Polytechnique Fédérale de Lausanne (EPFL), \\ CH-1015 Lausanne, Switzerland
}

(Received 31 August 2006; accepted 25 April 2007; published online 30 May 2007)

\begin{abstract}
Mass selected cations of $\mathrm{Ag}$ clusters are deposited, together with $\mathrm{Ar}$ seeded with $\mathrm{CO}_{2}$ (or other electron scavengers such as $\mathrm{O}_{2}, \mathrm{~N}_{2} \mathrm{O}$, or $\mathrm{CCl}_{4}$ ), on a cold substrate, while being flooded with low energy electrons. Optical absorption measurements reveal an efficient trapping of Ag cluster cations in the $\mathrm{Ar}$ matrix, provided that $\mathrm{CO}_{2}$ or another electron scavenger is present to ensure charge neutrality of the matrix. The spectrum of $\mathrm{Ag}_{3}^{+}$thus obtained is in excellent agreement with previous predictions from quantum chemical calculations. (C) 2007 American Institute of Physics.
\end{abstract}

[DOI: $10.1063 / 1.2741547]$

\section{INTRODUCTION}

Since the late 1970s much effort has been made to determine the electronic properties of small metal clusters. Noble metal clusters such as silver clusters are of special interest because the electronic properties are influenced by the nearby $d$ shell. On the one hand, neutral silver clusters embedded in rare gas matrices have been studied by absorption, fluorescence, and excitation spectroscopy. ${ }^{1-6}$ In all these studies the cluster cations are produced and mass selected in the gas phase, and then codeposited with a rare gas and an excess of low energy electrons for neutralization on a cold substrate to form a matrix seeded with monodispersed neutral clusters. On the other hand, the electronic structure and the optical properties of silver cluster cations ${ }^{7-10}$ and anions ${ }^{11}$ with up to 70 atoms have been investigated in the gas phase by photodepletion techniques.

A renewed interest in the optical activity of ions arose from the discovery of the luminescence of small silver clusters in the presence of an oxide where charge exchange occurs $^{12,13}$ and their optical activity when attached as markers to a biomolecule. ${ }^{14,15}$

While rare gas isolation studies present advantages in increasing the stability and density of the clusters and allowing a precise control of their temperature, the deposition of ions in pure rare gas matrices remains difficult since the space charge formed by the accumulation of the ions deflects any further incoming ions. This severely limits the density of matrix-isolated ions that can be attained in this way, making it difficult to use standard optical spectroscopy techniques to investigate the ions.

Such matrix isolation studies on ions were shown, however, to be possible with ion sources considerably more intense than our's, and the spectroscopic detection of mass selected matrix-isolated cations was first demonstrated by Leutwyler et al. ${ }^{16}$ This surprising observation was explained several years later by Godbout et al., ${ }^{17}$ who investigated the origin of the matrix stability by depositing $\mathrm{CO}_{2}^{+}$molecules in a neon matrix slightly doped with $\mathrm{CO}_{2}$ or $\mathrm{CCl}_{4}$. They inferred from their experiments that negative charges are produced by the collision of cations deflected on the side of the cryostat by the accumulated charge; these negative charges are trapped in the matrix in the form of $\mathrm{CO}_{2}^{-}$anions. Other electron acceptor molecules such as freons, ${ }^{18,19} \mathrm{CCl}_{4},{ }^{20,21}$ $\mathrm{CH}_{2} \mathrm{Cl}_{2},{ }^{22}$ or $\mathrm{N}_{2} \mathrm{O}$ (Ref. 23) have been used in matrix isolation photolysis and radiolysis experiments for a long time. In such studies a mixture of the molecules under investigation and of electron acceptor molecules is condensed in the rare gas matrix before irradiation. The electron scavengers trap the electrons resulting from the irradiation and therefore help stabilizing the cations in the matrix.

While the electron affinity of $\mathrm{CO}_{2}$ has been measured to be negative, equal to $-0.6 \mathrm{eV}$ in the gas phase, ${ }^{24}$ Takahashi et al. ${ }^{25}$ have shown that a solvent with a dielectric constant between 1.5 and 2 is sufficient to stabilize the additional electron. $\mathrm{CO}_{2}^{-}$is thus stable in an argon matrix that has a dielectric constant of 1.7 , as it has been observed in several experimental studies in $\operatorname{argon}^{21,26}$ and in neon. ${ }^{17,27,28}$

In the experiments that we performed up to now, the neutralization of the matrix is obtained by flooding the incoming cluster cations (typically one cation per $10^{5}$ matrix atoms) with low energy electrons. They attach to the cluster cations, neutralize the matrix, and thus allow to circumvent the space charge problem. In the present experiment we propose a simple method to accumulate cluster cations very efficiently in a rare gas matrix. Here, in order to circumvent the space charge limitation, an equivalent amount of anions and cations is introduced in the matrix. This is obtained by mixing to the matrix gas a small fraction of $\mathrm{CO}_{2}$ (typically $1 \%$ ), acting as an electron scavenger. By choosing a dilution of the impurity gas about three orders of magnitude higher than that of the incoming cluster cations, the low energy electrons are preferentially captured by the $\mathrm{CO}_{2}$ molecules for statistical reasons, leaving charged cluster cations in the matrix. The correct balance of the charges assuring the global neutrality of the matrix is automatically ensured if the number of low energy electrons is sufficient. Notice also that we have verified that $\mathrm{CO}_{2}$ has no optical activity in the near UV-visible range.

We demonstrate the efficiency of this method by measuring the first absorption spectrum for $\mathrm{Ag}_{3}^{+}$and compare our results to existing calculations by Bonačić-Koutecký et al. ${ }^{29}$ 


\section{EXPERIMENTAL SETUP}

The production and mass selection of positively charged silver clusters have been described in detail elsewhere. ${ }^{30}$ Silver ions are sputtered from a silver target and mass selected. The positively charged clusters are codeposited with $\mathrm{Ar}$ mixed with a small fraction of electron scavengers on a cold sample holder $(26 \mathrm{~K})$. Low energy electrons (about ten times more than the incoming clusters) are emitted from a shielded hot filament and flood the deposition region. Without these electrons a space charge builds up and most of the beam is deflected after a few seconds/minutes only.

The $\mathrm{Ag}_{3}^{+}$clusters with a current density of $9.5 \mathrm{nA} / \mathrm{cm}^{2}$ are accumulated in the matrix for $2.5 \mathrm{~h}$ at a deposition energy of $20 \mathrm{eV}$. According to molecular calculations, ${ }^{31}$ the very small clusters penetrate a few monolayers into the matrix. Electrons near the surface are then attracted by the positive charge and trapped on $\mathrm{CO}_{2}$ molecules. The matrix flux is calibrated such as to grow a $40 \mu \mathrm{m}$ thick matrix in that time, corresponding to the $1: 10^{5}$ ratio of $\mathrm{Ag}_{3}^{+}: \mathrm{Ar}$ mentioned above. After the deposition, a capping layer of argon is grown on top of the matrix to protect it from any contamination and to optimize the optical transmission of the matrix.

Optical absorption spectra are recorded through the lateral dimension of the matrix, i.e., perpendicular to the deposition axis. ${ }^{32}$ This geometry allows an enhanced sensitivity due to the long optical path. The light having crossed the matrix is collected with an optical fiber and analyzed by an optical spectrometer coupled to a liquid-nitrogen-cooled charge coupled device detector. Comparing this signal to the reference signal of a matrix with no clusters yields the absorption spectrum of the deposited species.

\section{RESULTS AND DISCUSSION}

Figure 1(a) shows the absorption spectrum measured after deposition of $\mathrm{Ag}_{3}^{+}$in a pure argon matrix, flooded with low energy electrons. The spectrum is in very good agreement with previously acquired absorption and excitation spectra ${ }^{2,4}$ on neutral $\mathrm{Ag}_{3}$ as well as with the calculated spectrum. ${ }^{29}$ In detail, the peaks at 2.39, 2.46, 3.16, 3.56, and $3.89 \mathrm{eV}$ are attributed to $\mathrm{Ag}_{3}$, while the smaller peaks at 3.94, 4.08, and $4.15 \mathrm{eV}$ are due to neutral $\mathrm{Ag}$ atoms. The peak at $2.82 \mathrm{eV}$ is tentatively assigned to neutral $\mathrm{Ag}_{2}{ }^{2}$ Both monomer and dimer result from the fragmentation occurring during the deposition process.

Figure 1(b) represents the absorption spectrum measured following the same deposition procedure but introducing $1 \%$ of $\mathrm{CO}_{2}$ as doping gas. There is a remarkable difference between the two spectra, indicating that we are studying the optical properties of two distinct species. While the spectrum in pure argon contains several narrow peaks at low energies, only a few small features are observable at the corresponding energies in the argon doped matrix, and the main transitions occur above $4 \mathrm{eV}$. Two novel and intense peaks are visible at 4.2 and $5.6 \mathrm{eV}$, while less intense peaks at 3.16 and $3.56 \mathrm{eV}$ are assigned to the neutral $\mathrm{Ag}_{3}$ and the peak at $3.94 \mathrm{eV}$ is due to neutral $\mathrm{Ag}$ resulting from fragmentation during the deposition process.

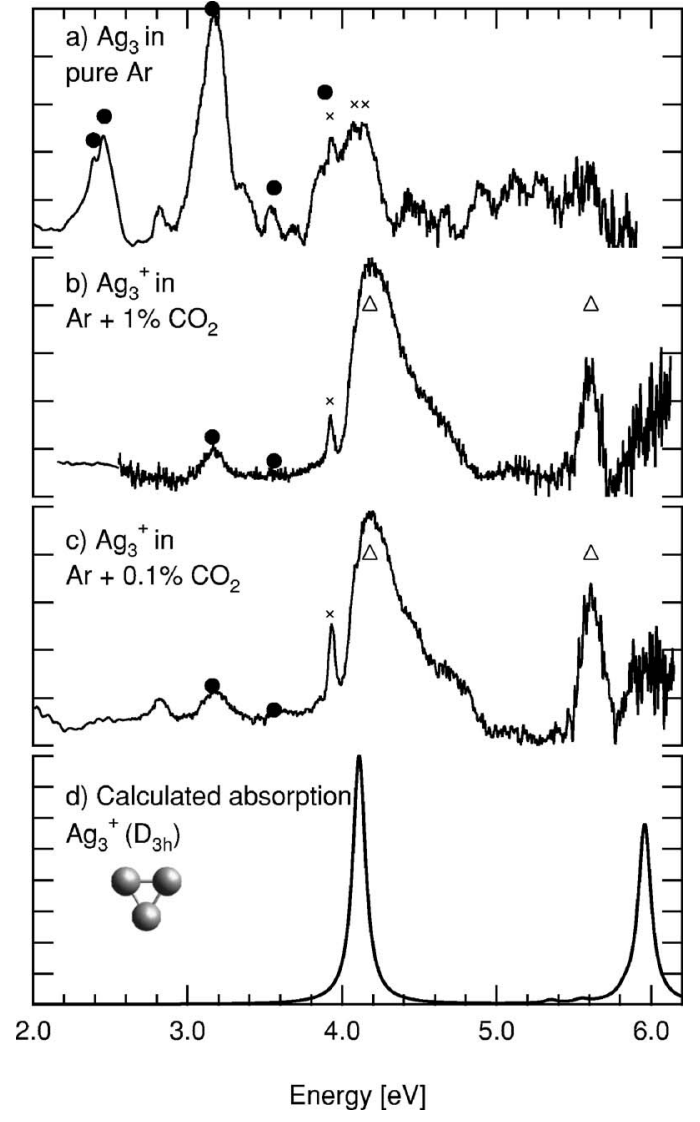

FIG. 1. Absorption spectra of $\mathrm{Ag}_{3}^{+}$at $26 \mathrm{~K}$ (a) in a pure argon matrix, (b) in an argon doped matrix with $1 \% \mathrm{CO}_{2}$, (c) in an argon matrix with $0.1 \% \mathrm{CO}_{2}$, and (d) calculated absorption spectrum of $\mathrm{Ag}_{3}^{+}$for the lowest energy isomer $D_{3 h}$ (Ref. 29) The peaks are marked as follows: $(\times) \mathrm{Ag}_{1},(\bigcirc) \mathrm{Ag}_{3}$, and $(\triangle)$ $\mathrm{Ag}_{3}^{+}$.

Since the absorption spectrum of $\mathrm{Ag}_{3}^{+}$has not been measured before, we have performed control experiments serving as indirect proof of the correct assignment of the two new peaks.

Notice first that the peaks resulting from fragmentation and corresponding to neutral $\mathrm{Ag}$ atoms and neutral $\mathrm{Ag}_{2}$ molecules correspond to their known absorption spectra. This is already an indication that the clusters are not altered by the doping gas. However, to give further evidence excluding a possible contamination of our spectra, we made the following experiments. First a matrix of Ar with $1 \%$ of $\mathrm{CO}_{2}$ with no clusters was grown and showed no optical activity, ruling out any effect due to isolated $\mathrm{CO}_{2}$ in the matrix. In a second experiment the deposition of Fig. 1(b) was repeated, but the concentration of the $\mathrm{CO}_{2}$ was reduced to $0.1 \%$. The resulting absorption spectrum is shown in Fig. 1(c). The main features at 4.2 and $5.6 \mathrm{eV}$ are identical as in Fig. 1(b), thus demonstrating that they are not due to some bonding of $\mathrm{Ag}_{3}$ with $\mathrm{CO}_{2}$, since if this were the case their intensity would have decreased significantly.

This conclusion is supported by other control experiments where $\mathrm{CO}_{2}$ was replaced by other electron scavengers such as $\mathrm{O}_{2}$ [Fig. 2(b)], $\mathrm{N}_{2} \mathrm{O}$ [Fig. 2(c)], and $\mathrm{CCl}_{4}$ [Fig. 2(d)]. The dominant peak at $4.2 \mathrm{eV}$ is visible in all four spectra, providing the experimental evidence that it is associated with the $\mathrm{Ag}_{3}$ cation. The second peak at $5.6 \mathrm{eV}$ is also clearly 


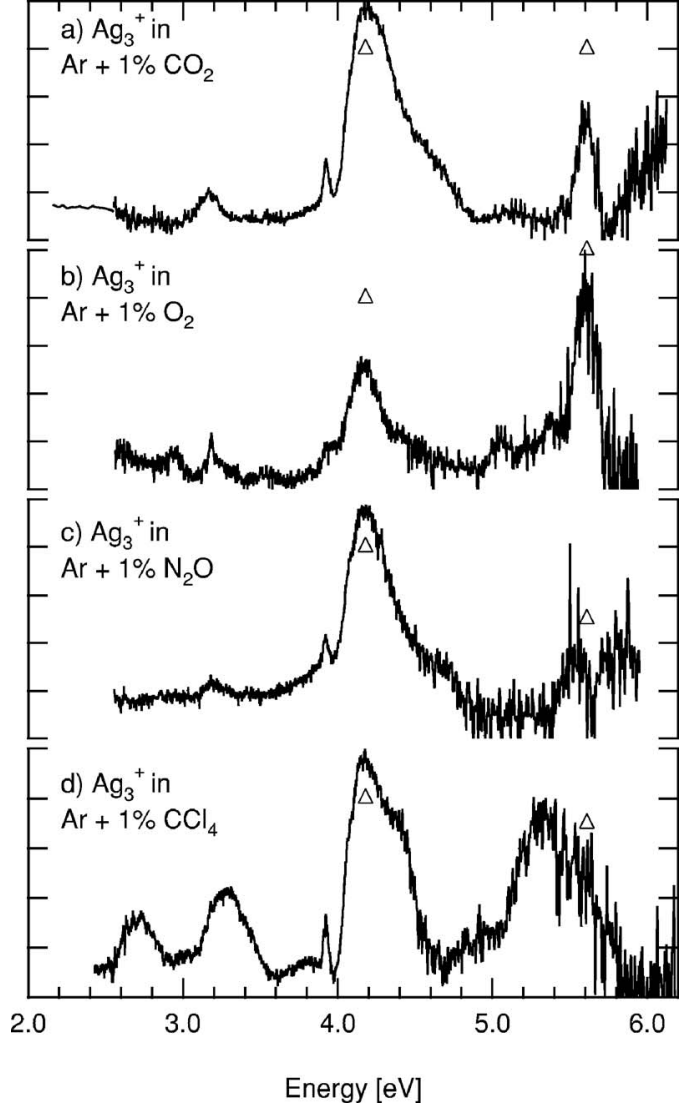

FIG. 2. Absorption spectra of $\mathrm{Ag}_{3}^{+}$at $26 \mathrm{~K}$ in a matrix doped with (a) $1 \%$ $\mathrm{CO}_{2}$, (b) $1 \% \mathrm{O}_{2}$, (c) $1 \% \mathrm{CO}_{2}$, and (d) $1 \% \mathrm{CCl}_{4}$. The peaks attributed to $\mathrm{Ag}_{3}^{+}$ are marked as $\triangle$.

visible with an $\mathrm{O}_{2}$ and $\mathrm{N}_{2} \mathrm{O}$ doping, although the optical transmission was reduced by one order of magnitude as compared to $1 \% \mathrm{CO}_{2}$. This induced a reduced signal to noise ratio as well as differences in the relative intensities of the two peaks discussed above, since the background substraction procedures were then more difficult. For $\mathrm{CCl}_{4}$ doping, the spectra show signatures of the presence of the doping molecules with the appearance of additional peaks that are not there with the other dopants. These peaks are either due to $\mathrm{CCl}_{4}^{-}$(Ref. 33) or to a more reactive interaction with the $\mathrm{Ag}_{3}^{+}$clusters. The high energy absorption peak at $5.6 \mathrm{eV}$ is therefore more difficult to observe since it is hidden in the side of a nearby absorption peak. It is worth mentioning that when the silver trimer is deposited in an argon matrix doped with $1 \% \mathrm{CO}$, the absorption spectrum disappears almost completely. This indicates that in this case there is a strong chemical interaction of the cluster with a $\mathrm{CO}$ molecule. It also indicates that a small cluster with a deposition energy of $20 \mathrm{eV}$ comes into contact with at least 100 gas atoms in the collision process, which is not surprising on the basis of known calculations. ${ }^{31}$ This suggests that for $\mathrm{CO}_{2}, \mathrm{O}_{2}, \mathrm{~N}_{2} \mathrm{O}$, and probably to a lesser extent for $\mathrm{CCl}_{4}$, the silver trimer cations have been in contact with the dopant molecule, without any significant effect on their optical signature.

It was suggested to us that the presence of $\mathrm{CO}_{2}^{-}$anions in the matrix could be revealed by a bleaching experiment. This is based on experiments performed by Thompson and Jacox, ${ }^{28}$ who observed a bleaching of the IR peaks attributed to $\mathrm{CO}_{2}^{-}$when irradiating their neon matrix with wavelengths longer than $695 \mathrm{~nm}$. Irradiation experiments with wavelengths longer than $695 \mathrm{~nm}$ have been performed on our samples. We observed that the spectral features disappeared slowly without significant changes of the shape of the spectra and, in particular, no increase of the neutral trimer spectra. We interpret this result as due to the thermal diffusion of the clusters in the matrix. Intense irradiation experiments accelerated this diffusion process, probably by a further local increase of the matrix temperature.

The structure and optical absorption spectrum of the silver trimer cation have been calculated by Bonačić-Koutecký et $a{ }^{29}$ The calculation gives a $D_{3 h}$ geometry for the most stable isomer in vacuum because of the absence of a JahnTeller effect; its calculated spectrum is reported in Fig. 1(e). The two observed transitions at 4.2 and $5.6 \mathrm{eV}$ are in good agreement with the calculated transitions at $4.11 \mathrm{eV}\left(1^{1} E^{\prime}\right.$ state with a calculated oscillator strength of 0.80$)$ and $5.96 \mathrm{eV}\left(2{ }^{1} A_{2}^{\prime \prime}\right.$ state with an oscillator strength of 0.48$)$. We therefore confidently assign the two peaks at 4.2 and $5.6 \mathrm{eV}$ to $\mathrm{Ag}_{3}^{+}$embedded in argon matrix. The calculations showed that while $d$ electrons are not explicitly involved in the intense transitions of $\mathrm{Ag}_{3}$, for the cation their role seems to be important for all intense transitions.

Notice that the observed peak at $4.2 \mathrm{eV}$ is blueshifted by $0.07 \mathrm{eV}$ compared to the calculated transition, whereas the peak at $5.6 \mathrm{eV}$ is redshifted by $0.35 \mathrm{eV}$. This shows that the effect of the matrix on the optical spectrum of very small clusters cannot be accounted for by a simple dielectric shift. $^{34,35}$

The amount of neutralized $\mathrm{Ag}_{3}^{+}$cluster ions can be estimated by comparing the absolute intensity of the peak at $3.16 \mathrm{eV}$ in Figs. 1(a)-1(c). We find a neutralization ratio of $\sim 15 \%$ for both $1 \%$ and $0.1 \% \mathrm{CO}_{2}$. This is more than the ratio of cluster ions to $\mathrm{CO}_{2}$ molecules that is expected on pure statistical arguments. However, this relatively high value might be explained by the electric force that attracts the electrons to the cations when they still lie on or close to the surface.

\section{CONCLUSION}

We applied an efficient method for embedding cations in a rare gas matrix slightly doped with $\mathrm{CO}_{2}$. In the resulting matrix the $\mathrm{CO}_{2}$ molecules act as electron scavengers; their concentration is set to be orders of magnitude more abundant than the clusters but also orders of magnitude less abundant than argon. The low energy electrons provided for the neutralization of the clusters attach preferentially to the electron scavengers for statistical reasons and the matrix formed is composed of an equal number of positive and negative ions. We use this method to record the absorption spectrum of $\mathrm{Ag}_{3}^{+}$, this is to our knowledge the first absorption spectrum of this cation. The spectrum is found to be in excellent agreement with theory. A further experimental proof for the trapping of the ions comes from the comparison of the spectra recorded with other electron scavengers. $\mathrm{CO}_{2}$ is found to be an ideal dopant for there is no optical activity in the visible range for the molecule and its anion; it is inert and does not 
react with the cluster and it additionally provides the best optical transmission of the matrix. The efficiency of cation stabilization is estimated to $85 \%$.

\section{ACKNOWLEDGMENT}

The authors would like to thank Professor Thomas Bally for a valuable discussion on the stabilization of ions in rare gas matrices.

${ }^{1}$ W. Harbich, S. Fedrigo, and J. Buttet, Chem. Phys. Lett. 195, 5 (1992).

${ }^{2}$ S. Fedrigo, W. Harbich, and J. Buttet, J. Chem. Phys. 99, 5712 (1993).

${ }^{3}$ C. Félix, C. Sieber, W. Harbich, J. Buttet, I. Rabin, W. Schulze, and G. Ertl, Chem. Phys. Lett. 313, 105 (1999).

${ }^{4}$ I. Rabin, W. Schulze, G. Ertl, C. Félix, C. Sieber, W. Harbich, and J. Buttet, Chem. Phys. Lett. 320, 59 (2000).

${ }^{5}$ C. Félix, C. Sieber, W. Harbich, J. Buttet, I. Rabin, W. Schulze, and G. Ertl, Phys. Rev. Lett. 86, 2992 (2001).

${ }^{6}$ C. Sieber, W. Harbich, J. Buttet, C. Félix, R. Mitrić, and V. BonačićKoutecký, Phys. Rev. A 70, 041201 (2004).

${ }^{7}$ J. Tiggesbaumker, L. Koller, H. O. Lutz, and K. H. Meiwes Broer, Chem. Phys. Lett. 190, 42 (1992).

${ }^{8}$ J. Tiggesbaumker, L. Koller, K. H. Meiwes Broer, and A. Liebsch, Phys. Rev. A 48, R1749 (1993).

${ }^{9}$ D. Schooss, S. Gilb, J. Kaller, M. M. Kappes, F. Furche, A. Kohn, K. May, and R. Ahlrichs, J. Chem. Phys. 113, 5361 (2000).

${ }^{10}$ A. Schweizer, J. M. Weber, S. Gilb, H. Schneider, D. Schooss, and M. M. Kappes, J. Chem. Phys. 119, 3699 (2003).

${ }^{11}$ J. Tiggesbaumker, L. Koller, and K. H. Meiwes Broer, Chem. Phys. Lett. 260, 3 (1996)

${ }^{12}$ L. A. Peyser, A. E. Vinson, A. P. Bartko, and R. M. Dickson, Science 291, 103 (2001).

${ }^{13}$ C. Bürgel, R. Mitrić, and V. Bonačić-Koutecký, Appl. Phys. A: Mater. Sci. Process. 82, 117 (2006)

${ }^{14}$ R. Antoine, T. Tabarin, M. Broyer, P. Dugourd, R. Mitrić, and V.
Bonačić-Koutecký, ChemPhysChem 7, 524 (2006).

${ }^{15}$ I. Compagnon, T. Tabarin, R. Antoine, M. Broyer, P. Dugourd, R. Mitric, J. Petersen, and V. Bonacic-Koutecky, J. Chem. Phys. 125, 164326 (2006)

${ }^{16}$ S. Leutwyler, J. P. Maier, and U. Spittel, Chem. Phys. Lett. 96, 645 (1983).

${ }^{17}$ J. Godbout, T. Halasinski, G. Leroi, and J. Allison, J. Phys. Chem. 100, 2892 (1996)

${ }^{18}$ F. T. Prochaska and L. Andrews, J. Chem. Phys. 68, 5577 (1978).

${ }^{19}$ V. I. Feldman, Radiat. Phys. Chem. 55, 565 (1999).

${ }^{20}$ L. Andrews, B. J. Kelsall, J. H. Miller, and B. W. Keelan, J. Chem. Soc., Faraday Trans. 2 79, 1417 (1983)

${ }^{21}$ M. Zhou and L. Andrews, J. Chem. Phys. 110, 2414 (1999).

${ }^{22}$ W. Tang, X.-L. Zhang, and T. Bally, J. Phys. Chem. 97, 4373 (1993).

${ }^{23}$ I. Shnitko, J. Fulara, A. Batalov, C. Gillery, H. Masso, P. Rosmus, and J. Maier, J. Phys. Chem. A 110, 2885 (2006).

${ }^{24}$ R. N. Compton, P. W. Reinhardt, and C. D. Cooper, J. Chem. Phys. 63, 3821 (1975).

${ }^{25}$ K. Takahashi, S. Sawamura, N. M. Dimitrijevic, D. M. Bartels, and C. D. Jonah, J. Phys. Chem. A 106, 108 (2002).

${ }^{26} \mathrm{M}$. Zhou and L. Andrews (Ref. 21) have shown that in argon matrices above $25 \mathrm{~K}$, the $\mathrm{CO}_{2}^{-}$can react with a $\mathrm{CO}_{2}$ molecule to form a symmetrical $\mathrm{C}_{2} \mathrm{O}_{4}^{-}$anion.

${ }^{27}$ M. E. Jacox and W. E. Thompson, J. Chem. Phys. 91, 1410 (1989).

${ }^{28}$ W. E. Thompson and M. E. Jacox, J. Chem. Phys. 111, 4487 (1999).

${ }^{29}$ V. Bonačić-Koutecký, J. Pittner, M. Boiron, and P. Fantucci, J. Chem. Phys. 110, 3876 (1999).

${ }^{30}$ P. Fayet and L. Wöste, Z. Phys. D: At., Mol. Clusters 3, 177 (1986).

${ }^{31}$ M. Ratner, W. Harbich, and S. Fedrigo, Phys. Rev. B 60, 11730 (1999).

${ }^{32}$ F. Conus, J. T. Lau, V. Rodrigues, and C. Félix, Rev. Sci. Instrum. 77, 113103 (2006).

${ }^{33}$ H. Muto and K. Nunome, J. Chem. Phys. 94, 4741 (1991).

${ }^{34}$ B. Gervais, E. Giglio, E. Jacquet, A. Ipatov, P.-G. Reinhard, F. Fehrer, and E. Suraud, Phys. Rev. A 71, 015201 (2005).

${ }^{35}$ F. Conus, V. Rodrigues, S. Lecoultre, A. Rydlo, and C. Félix, J. Chem. Phys. 125, 024511 (2006). 